\title{
A Static Analyzer for Large Safety-Critical Software
}

\author{
(Extended Abstract)
}

\author{
Bruno Blanchet $* \S$ \\ Laurent Mauborgne ${ }^{\S}$
}

\author{
Patrick Cousot ${ }^{\S}$ \\ Antoine Miné ${ }^{\S}$
}

\author{
Radhia Cousot*a \\ David Monniaux*\$
}

\author{
Jérôme Feret ${ }^{\S}$ \\ Xavier Rival ${ }^{\S}$
}

\begin{abstract}
We show that abstract interpretation-based static program analysis can be made efficient and precise enough to formally verify a class of properties for a family of large programs with few or no false alarms. This is achieved by refinement of a general purpose static analyzer and later adaptation to particular programs of the family by the end-user through parametrization. This is applied to the proof of soundness of data manipulation operations at the machine level for periodic synchronous safety critical embedded software.

The main novelties are the design principle of static analyzers by refinement and adaptation through parametrization (Sect. 3 and 7), the symbolic manipulation of expressions to improve the precision of abstract transfer functions (Sect. 6.3), the octagon (Sect. 6.2.2), ellipsoid (Sect. 6.2.3), and decision tree (Sect. 6.2.4) abstract domains, all with sound handling of rounding errors in floating point computations, widening strategies (with thresholds: Sect. 7.1.2, delayed: Sect. 7.1.3) and the automatic determination of the parameters (parametrized packing: Sect. 7.2).
\end{abstract}

\section{Categories and Subject Descriptors}

D.2.4 [Software Engineering]: Program Verification-formal methods, validation, assertion checkers; D.3.1 [Programming Languages]: Formal Definitions and Theory-semantics; F.3.1 [Logics and Meanings of Programs]: Specifying and Verifying and Reasoning about ProgramsMechanical verification, assertions, invariants; F.3.2 [Logics and Meanings of Programs]: Semantics of Programming Languages-Denotational semantics, Program analysis.

\section{General Terms}

Algorithms, Design, Experimentation, Reliability, Theory, Verification.

\section{Keywords}

Abstract Interpretation; Abstract Domains; Static Analysis; Verification; Floating Point; Embedded, Reactive, RealTime, Safety-Critical Software.

\footnotetext{
* CNRS (Centre National de la Recherche Scientifique)

$\S$ École normale supérieure. First-name.Last-name@ens.fr

I École polytechnique. First-name.Last-name@polytechnique.fr Permission to make digital or hard copies of all or part of this work for personal or classroom use is granted without fee provided that copies are not made or distributed for profit or commercial advantage and that copies bear this notice and the full citation on the first page. To copy otherwise, to republish, to post on servers or to redistribute to lists, requires prior specific permission and/or a fee.
}

PLDI'03, June 9-11, 2003, San Diego, California, USA.

Copyright 2003 ACM 1-58113-662-5/03/0006 ...\$5.00.

\section{INTRODUCTION}

Critical software systems (as found in industrial plants, automotive, and aerospace applications) should never fail. Ensuring that such software does not fail is usually done by testing, which is expensive for complex systems with high reliability requirements, and anyway fails to prove the impossibility of failure. Formal methods, such as model checking, theorem proving, and static analysis, can help.

The definition of "failure" itself is difficult, in particular in the absence of a formal specification. In this paper, we choose to focus on a particular aspect found in all specifications for critical software, that is, ensuring that the critical software never executes an instruction with "undefined" or "fatal error" behavior, such as out-of-bounds accesses to arrays or improper arithmetic operations (such as overflows or division by zero). Such conditions ensure that the program is written according to its intended semantics, for example the critical system will never abort its execution. These correctness conditions are automatically extractable from the source code, thus avoiding the need for a costly formal specification. Our goal is to prove automatically that the software never executes such erroneous instructions or, at least, to give a very small list of program points that may possibly behave in undesirable ways.

In this paper, we describe our implementation and experimental studies of static analysis by abstract interpretation over a family of critical software systems, and we discuss the main technical choices and possible improvements.

\section{REQUIREMENTS}

When dealing with undecidable questions on program execution, the verification problem must reconcile correctness (which excludes non exhaustive methods such as simulation or test), automation (which excludes model checking with manual production of a program model and deductive methods where provers must be manually assisted), precision (which excludes general analyzers which would produce too many false alarms, i.e., spurious warnings about potential errors), scalability (for software of a few hundred thousand lines), and efficiency (with minimal space and time requirements allowing for rapid verification during the software production process which excludes a costly iterative refinement process).

Industrialized general-purpose static analyzers satisfy all criteria but precision and efficiency. Traditionally, static analysis is made efficient by allowing correct but somewhat imprecise answers to undecidable questions. In many usage contexts, imprecision is acceptable provided all answers are 
sound and the imprecision rate remains low (e.g. 5 to $15 \%$ of the runtime tests cannot typically be eliminated). This is the case for program optimization (such as static elimination of run-time array bound checks), program transformation (such as partial evaluation), etc.

In the context of program verification, where human interaction must be reduced to a strict minimum, false alarms are undesirable. A $5 \%$ rate of false alarms on a program of a few hundred thousand lines would require a several personyear effort to manually prove that no error is possible. Fortunately, abstract interpretation theory shows that for any finite class of programs, it is possible to achieve full precision and great efficiency [7] by discovering an appropriate abstract domain. The challenge is to show that this theoretical result can be made practical by considering infinite but specific classes of programs and properties to get efficient analyzers producing few or no false alarms. A first experiment on smaller programs of a few thousand lines was quite encouraging [5] and the purpose of this paper is to report on a real-life application showing that the approach does scale up.

\section{DESIGN PRINCIPLE}

The problem is to find an abstract domain that yields an efficient and precise static analyzer for the given family of programs. Our approach is in two phases, an initial design phase by specialists in charge of designing a parametrizable analyzer followed by an adaptation phase by end-users in charge of adapting the analyzer for (existing and future) programs in the considered family by an appropriate choice of the parameters of the abstract domain and the iteration strategy (maybe using some parameter adaptation strategies provided by the analyser).

\subsection{Initial Design by Refinement}

Starting from an existing analyzer [5], the initial design phase is an iterative manual refinement of the analyzer. We have chosen to start from a program in the considered family that has been running for 10 years without any run-time error, so that all alarms are, in principle, due to the imprecision of the analysis. The analyzer can thus be iteratively refined for this example until all alarms are eliminated.

Each refinement step starts with a static analysis of the program, which yields false alarms. Then a manual backward inspection of the program starting from sample false alarms leads to the understanding of the origin of the imprecision of the analysis. There can be two different reasons for the lack of precision:

- Some local invariants are expressible in the current version of the abstract domain but were missed either:

- because some abstract transfer function (Sect. 5.4) was too coarse, in which case it must be rewritten closer to the best abstraction of the concrete transfer function [9], (Sect. 6.3);

- or because a widening (Sect. 5.5) was too coarse, in which case the iteration strategy must be refined (Sect. 7.1);

- Some local invariants are necessary in the correctness proof but are not expressible in the current version of the abstract domain. To express these local invariants, a new abstract domain has to be designed by specialists and incorporated in the analyzer as an approximation of the reduced product [9] of this new component with the already existing domain (Sect. 7.2).
When this new refinement of the analyzer has been implemented, it is tested on typical examples and then on the full program to verify that some false alarms have been eliminated. In general the same cause of imprecision appears several times in the program; furthermore, one single cause of imprecision at some program point often leads later to many false alarms in the code reachable from that program point, so a single refinement typically eliminates a few dozen if not hundreds of false alarms.

This process is to be repeated until there is no or very few false alarms left.

\subsection{Adaptation by Parametrization}

The analyzer can then be used by end-users in charge of proving programs in the family. The necessary adaptation of the analyzer to a particular program in the family is by appropriate choice of some parameters. An example provided in the preliminary experience [5] was the widening with thresholds (Sect. 7.1.2). Another example is relational domains (such as octagons [30], Sect. 6.2.2) which cannot be applied to all global variables simultaneously because the corresponding analysis would be too expensive; it is possible to have the user supply for each program point groups of variables on which the relational analysis should be independently applied.

In practice we have discovered that the parametrization can be largely automated (and indeed it is fully automated for octagons as explained in Sect. 7). This way the effort to manually adapt the analyzer to a particular program in the family is reduced to a minimum.

\subsection{Analysis of the Alarms}

We implemented and used a slicer [34] to help in the alarm inspection process. If the slicing criterion is an alarm point, the extracted slice contains the computations that led to the alarm. However, the classical data and control dependence-based backward slicing turned out to yield prohibitively large slices.

In practice we are not interested in the computation of the variables for which the analyzer already provides a value close to end-user specifications, and we can consider only the variables we lack information about (integer or floating point variables that may contain large values or boolean variables that may take any value according to the invariant). In the future we plan to design more adapted forms of slicing: an abstract slice would only contain the computations that lead to an alarm point wherever the invariant is too weak.

\section{THE CONSIDERED FAMILY OF PRO- GRAMS}

The considered programs in the family are automatically generated using a proprietary tool from a high-level specification familiar to control engineers, such as systems of differential equations or synchronous operator networks (block diagrams as illustrated in Fig. 1), which is equivalent to the use of synchronous languages (like Lustre [20]). Such synchronous data-flow specifications are quite common in real-world safety-critical control systems ranging from letter sorting machine control to safety control and monitoring systems for nuclear plants and "fly-by-wire" systems. Periodic synchronous programming perfectly matches the need for the real-time integration of differential equations by for- 
ward, fixed step numerical methods. Periodic synchronous programs have the form:

declare volatile input, state and output variables; initialize state variables;

loop forever

- read volatile input variables,

- compute output and state variables,

- write to volatile output variables;

wait for next clock tick; end loop

Our analysis proves that no exception can be raised (but the clock tick) and that all data manipulation operations are sound. The bounded execution time of the loop body should also be checked by static analysis [16] to prove that the real-time clock interrupt does occur at idle time.

We operate on the $\mathrm{C}$ language source code of those systems, ranging from a few thousand lines to 132,000 lines of C source code $(75 \mathrm{kLOC}$ after preprocessing and simplification as in Sect. 5.1). We take into account all machinedependent aspects of the semantics of $\mathrm{C}$ (as described in [5]) as well as the periodic synchronous programming aspects (for the wait). We use additional specifications to describe the material environment with which the software interacts (essentially ranges of values for a few hardware registers containing volatile input variables and a maximal execution time to limit the possible number of iterations in the external loop ${ }^{1}$ ).

The source codes we consider use only a reduced subset of $\mathrm{C}$, both in the automatically generated glue code and the handwritten pieces. As it is often the case with critical systems, there is no dynamic memory allocation and the use of pointers is restricted to call-by-reference. On the other hand, an important characteristics of those programs is that the number of global and static ${ }^{2}$ variables is roughly linear in the length of the code. Moreover the analysis must consider the values of all variables and the abstraction cannot ignore any part of the program without generating false alarms. It was therefore a grand challenge to design an analysis that is precise and does scale up.

\section{STRUCTURE OF THE ANALYZER}

The analyzer is implemented in Objective Caml [25]. It operates in two phases: the preprocessing and parsing phase followed by the analysis phase.

\subsection{Preprocessing Phase}

The source code is first preprocessed using a standard C preprocessor, then parsed using a C99-compatible parser. Optionally, a simple linker allows programs consisting of several source files to be processed.

The program is then type-checked and compiled to an intermediate representation, a simplified version of the abstract syntax tree with all types explicit and variables given unique identifiers. Unsupported constructs are rejected at this point with an error message.

Syntactically constant expressions are evaluated and re-

\footnotetext{
${ }^{1}$ Most physical systems cannot run forever and some event counters in their control programs are bounded because of this physical limitation.

${ }^{2}$ In C, a static variable has limited lexical scope yet is persistent with program lifetime. Semantically, it is the same as a global variable with a fresh name.
}

placed by their value. Unused global variables are then deleted. This phase is important since the analyzed programs use large arrays representing hardware features with constant subscripts; those arrays are thus optimized away.

Finally the preprocessing phase includes preparatory work for trace partitioning (Sect. 7.1.5) and parametrized packing (Sect. 7.2).

\subsection{Analysis Phase}

The analysis phase computes the reachable states in the considered abstract domain. This abstraction is formalized by a concretization function $\gamma[8,9,11]$. The computation of the abstraction of the reachable states by the abstract interpreter is called abstract execution.

The abstract interpreter first creates the global and static variables of the program (the stack-allocated variables are created and destroyed on-the-fly). Then the abstract execution is performed compositionally, by induction on the abstract syntax, and driven by the iterator.

\subsection{General Structure of the Iterator}

The abstract execution starts at a user-supplied entry point for the program, such as the main function. Each program construct is then interpreted by the iterator according to the semantics of $\mathrm{C}$ as well as some information about the target environment (some orders of evaluation left unspecified by the $\mathrm{C}$ norm, the sizes of the arithmetic types, etc., see [5]). The iterator transforms the $\mathrm{C}$ instructions into directives for the abstract domain that represents the memory state of the program (Sect. 6.1), that is, the global, static and stack-allocated variables.

The iterator operates in two modes: the iteration mode and the checking mode. The iteration mode is used to generate invariants; no warning is displayed when some possible errors are detected. When in checking mode, the iterator issues a warning for each operator application that may give an error on the concrete level (that is to say, the program may be interrupted, such as when dividing by zero, or the computed result may not obey the end-user specification for this operator, such as when integers wrap-around due to an overflow). In all cases, the analysis goes on with the nonerroneous concrete results (overflowing integers are wiped out and not considered modulo, thus following the end-user intended semantics).

Tracing facilities with various degrees of detail are also available. For example the loop invariants which are generated by the analyzer can be saved for examination.

\subsection{Primitives of the Iterator}

Whether in iteration or checking mode, the iterator starts with an abstract environment $E^{\sharp}$ at the beginning of a statement $S$ in the program and outputs an abstract environment $\llbracket S \rrbracket^{\sharp}\left(E^{\sharp}\right)$ which is a valid abstraction after execution of statement $S$. This means that if a concrete environment maps variables to their values, $\llbracket S \rrbracket^{s}$ is a standard semantics of $S$ (mapping an environment $\rho$ before executing $S$ to the corresponding environment $\llbracket S \rrbracket^{s}(\rho)$ after execution of $S$ ), $\llbracket S \rrbracket^{c}$ is the collecting semantics of $S$ (mapping a set $E$ of environments before executing $S$ to the corresponding set $\llbracket S \rrbracket^{c}(E)=\left\{\llbracket S \rrbracket^{s}(\rho) \mid \rho \in E\right\}$ of environments after execution of $S), \gamma\left(E^{\sharp}\right)$ is the set of concrete environments before $S$ then $\llbracket S \rrbracket^{\sharp}\left(E^{\sharp}\right)$ over-approximates the set $\llbracket S \rrbracket^{c}\left(\gamma\left(E^{\sharp}\right)\right)$ of environments after executing $S$ in that $\llbracket S \rrbracket^{c}\left(\gamma\left(E^{\sharp}\right)\right) \subseteq$ 
$\gamma\left(\llbracket S \rrbracket^{\sharp}\left(E^{\sharp}\right)\right)$. The abstract semantics $\llbracket S \rrbracket^{\sharp}$ is defined as follows:

- Tests: let us consider a conditional

$$
S=\text { if }(c)\left\{S_{1}\right\} \text { else }\left\{S_{2}\right\}
$$

(an absent else branch is considered as an empty execution sequence). The condition $c$ can be assumed to have no side effect and to contain no function call, both of which can be handled by first performing a program transformation. The iterator computes:

$$
\llbracket S \rrbracket^{\sharp}\left(E^{\sharp}\right)=\llbracket S_{1} \rrbracket^{\sharp}\left(\operatorname{guard}^{\sharp}\left(E^{\sharp}, c\right)\right) \sqcup^{\sharp} \llbracket S_{2} \rrbracket^{\sharp}\left(\operatorname{guard}^{\sharp}\left(E^{\sharp}, \neg c\right)\right)
$$

where the abstract domain implements:

- $\sqcup^{\sharp}$ as the abstract union that is an abstraction of the union $\cup$ of sets of environments;

$-\operatorname{guard}^{\sharp}\left(E^{\sharp}, c\right)$ as an approximation of $\llbracket c \rrbracket^{c}\left(\gamma\left(E^{\sharp}\right)\right)$ where the collecting semantics $\llbracket c \rrbracket^{c}(E)=\left\{\rho \in E \mid \llbracket c \rrbracket^{s}(\rho)=\right.$ true $\}$ of the condition $c$ is the set of concrete environments $\rho$ in $E$ satisfying condition $c$. In practice, the abstract domain only implements guard ${ }^{\sharp}$ for atomic conditions and compound ones are handled by structural induction.

- Loops are by far the most delicate construct to analyze. Let us denote by $E_{0}^{\sharp}$ the environment before the loop:

$$
\text { while }(c)\{\text { body }\}
$$

The abstract loop invariant to be computed for the head of the loop is an upper approximation of the least invariant of $F$ where $F(E)=\gamma\left(E_{0}^{\sharp}\right) \cup \llbracket b o d y \rrbracket^{c}\left(\llbracket c \rrbracket^{c}(E)\right)$. The fixpoint computation $F^{\sharp}\left(E^{\sharp}\right)=E_{0}^{\sharp} \sqcup^{\sharp} \llbracket b o d y \rrbracket^{\sharp}\left(\operatorname{guard}^{\sharp}\left(E^{\sharp}, c\right)\right)$ is always done in iteration mode, requires a widening (Sect. 5.5) and stops with an abstract invariant $E^{\sharp}$ satisfying $F^{\sharp}\left(E^{\sharp}\right)$ $\sqsubseteq^{\sharp} E^{\sharp}$ (where the abstract partial ordering $x \sqsubseteq^{\sharp} y$ implies $\gamma(x) \subseteq \gamma(y))[11]$. When in checking mode, the abstract loop invariant has first to be computed in iteration mode and then, an extra iteration (in checking mode this time), starting from this abstract invariant is necessary to collect potential errors.

- Sequences $i_{1} ; i_{2}$ : first $i_{1}$ is analyzed, then $i_{2}$, so that:

$$
\llbracket i_{1} ; i_{2} \rrbracket^{\sharp}\left(E^{\sharp}\right)=\llbracket i_{2} \rrbracket^{\sharp} \circ \llbracket i_{1} \rrbracket^{\sharp}\left(E^{\sharp}\right) .
$$

- Function calls are analyzed by abstract execution of the function body in the context of the point of call, creating temporary variables for the parameters and the return value. Since the considered programs do not use recursion, this gives a context-sensitive polyvariant analysis semantically equivalent to inlining.

- Assignments are passed to the abstract domain.

- Return statement: We implemented the return statement by carrying over an abstract environment representing the accumulated return values (and environments, if the function has side effects).

\subsection{Least Fixpoint Approximation with Widening and Narrowing}

The analysis of loops involves the iterative computation of an invariant $E^{\sharp}$ that is such that $F^{\sharp}\left(E^{\sharp}\right) \sqsubseteq^{\sharp} E^{\sharp}$ where $F^{\sharp}$ is an abstraction of the concrete monotonic transfer function $F$ of the test and loop body. In abstract domains with infinite height, this is done by widening iterations computing a finite sequence $E_{0}^{\sharp}=\perp, \ldots, E_{n+1}^{\sharp}=E_{n}^{\sharp} \nabla F^{\sharp}\left(E_{n}^{\sharp}\right), \ldots$, $E_{N}^{\sharp}$ of successive abstract elements, until finding an invariant $E_{N}^{\sharp}$. The widening operator $\nabla$ should be sound (that is the concretization of $x \nabla y$ should overapproximate the concretizations of $x$ and $y$ ) and ensure the termination in finite time [8, 11] (see an example in Sect. 7.1.2).

In general, this invariant is not the strongest one in the abstract domain. This invariant is then made more and more precise by narrowing iterations: $E_{N}^{\sharp}, \ldots, E_{n+1}^{\sharp}=$ $E_{n}^{\sharp} \Delta F^{\sharp}\left(E_{n}^{\sharp}\right)$ where the narrowing operator $\Delta$ is sound (the concretization of $x \Delta y$ is an upper approximation of the intersection of $x$ and $y$ ) and ensures termination [8, 11].

\section{ABSTRACT DOMAINS}

The elements of an abstract domain abstract concrete predicates, that is, properties or sets of program states. The operations of an abstract domain are transfer functions abstracting predicate transformers corresponding to all basic operations in the program [8]. The analyzer is fully parametric in the abstract domain (this is implemented using an Objective Caml functor). Presently the analyzer uses the memory abstract domain of Sect. 6.1, which abstracts sets of program data states containing data structures such as simple variables, arrays and records. This abstract domain is itself parametric in the arithmetic abstract domains (Sect. 6.2) abstracting properties of sets of (tuples of) boolean, integer or floating-point values. Finally, the precision of the abstract transfer functions can be significantly improved thanks to symbolic manipulations of the program expressions preserving the soundness of their abstract semantics (Sect. 6.3).

\subsection{The Memory Abstract Domain}

When a $\mathrm{C}$ program is executed, all data structures (simple variables, arrays, records, etc) are mapped to a collection of memory cells containing concrete values. The memory abstract domain is an abstraction of sets of such concrete memory states. Its elements, called abstract environments, map variables to abstract cells. The arithmetic abstract domains operate on the abstract value of one cell for non-relational ones (Sect. 6.2.1) and on several abstract cells for relational ones (Sect. 6.2.2, 6.2.3, and 6.2.4). An abstract value in a abstract cell is therefore the reduction of the abstract values provided by each different basic abstract domain (that is an approximation of their reduced product [9]).

\subsubsection{Abstract Environments}

An abstract environment is a collection of abstract cells, which can be of the following four types:

- An atomic cell represents a variable of a simple type (enumeration, integer, or float) by an element of the arithmetic abstract domain. Enumeration types, including the booleans, are considered to be integers.

- An expanded array cell represents a program array using one cell for each element of the array. Formally, let $A=\left(\left(v_{1}^{i}, \ldots, v_{n}^{i}\right)\right)_{i \in \Delta}$ be the family (indexed by a set $\Delta$ ) of values of the array (of size $n$ ) to be abstracted. The abstraction is $\perp$ (representing non-accessibility of dead code) when $A$ is empty. Otherwise the abstraction is an abstract array $A_{e}^{\sharp}$ of size $n$ such the expanded array cell $A_{e}^{\sharp}[k]$ is the abstraction of $\bigcup_{i \in \Delta} v_{k}^{i}$ for $k=1, \ldots, n$. Therefore the abstraction is component-wise, each element of the array being abstracted separately.

- A shrunk array cell represents a program array using a single cell. Formally the abstraction is a shrunk array cell $A_{s}^{\sharp}$ abstracting $\bigcup_{k=1}^{n} \bigcup_{i \in \Delta} v_{k}^{i}$. All elements of the array are thus "shrunk" together. We use this representation for large arrays where all that matters is the range of the stored data. 
- A record cell represents a program record (struct) using one cell for each field of the record. Thus our abstraction is field-sensitive.

\subsubsection{Fast Implementation of Abstract Environments}

A naive implementation of abstract environments may use an array. We experimented with in-place and functional arrays and found this approach very slow. The main reason is that abstract union $\sqcup^{\sharp}$ operations are expensive, because they operate in time linear in the number of abstract cells; since both the number of global variables (whence of abstract cells) and the number of tests (involving the abstract union $\left.\sqcup^{\sharp}\right)$ are linear in the length of the code, this yields a quadratic time behavior.

A simple yet interesting remark is that in most cases, abstract union operations are applied between abstract environments that are identical on almost all abstract cells: branches of tests modify a few abstract cells only. It is therefore desirable that those operations should have a complexity proportional to the number of differing cells between both abstract environments. We chose to implement abstract environments using functional maps implemented as sharable balanced binary trees, with short-cut evaluation when computing the abstract union, abstract intersection, widening or narrowing of physically identical subtrees [5, $\S 6.2]$. An additional benefit of sharing is that it contributes to the rather light memory consumption of our analyzer.

On a 10,000-line example we tried [5], the execution time was divided by seven, and we are confident that the execution times would have been prohibitive for the longer examples. The efficiency of functional maps in the context of sophisticated static analyses has also been observed by [26] for representing first-order structures.

\subsubsection{Operations on Abstract Environments}

Operations on a $\mathrm{C}$ data structure are translated into operations on cells of the current abstract environments. Most translations are straightforward.

- Assignments: In general, an assignment lvalue $:=e$ is translated into the assignment of the abstract value of $e$ into the abstract cell corresponding to lvalue. However, for array assignments, such as $x[i]:=e$, one has to note that the array index $i$ may not be fully known, so all cells possibly corresponding to $x[i]$ may either be assigned the value of $e$, or keep their old value. In the analysis, these cells are assigned the upper bound of their old abstract value and the abstract value of $e$. Similarly, for a shrunk array $x$, after an assignment $x[i]:=e$, the cell representing $x$ may contain either its old value (for array elements not modified by the assignment), or the value of $e$.

- Guard: The translation of concrete to abstract guards is not detailed since similar to the above case of assignments.

- Abstract union, widening, narrowing: Performed cellwise between abstract environments.

\subsection{Arithmetic Abstract Domains}

The non-relational arithmetic abstract domains abstract sets of numbers while the relational domains abstract sets of tuples of numbers. The basic abstract domains we started with [5] are the intervals and the clocked abstract domain abstracting time. They had to be significantly refined using octagons (Sect. 6.2.2), ellipsoids (Sect. 6.2.3) and decision trees (Sect. 6.2.4).

\subsubsection{Basic Abstract Domains}

- The Interval Abstract Domain. The first, and simplest, implemented domain is the domain of intervals, for both integer and floating-point values [8]. Special care has to be taken in the case of floating-point values and operations to always perform rounding in the right direction and to handle special IEEE [23] values such as infinities and NaNs (Not a Number).

- The Clocked Abstract Domain. A simple analysis using the intervals gives a large number of false warnings. A great number of those warnings originate from possible overflows in counters triggered by external events. Such errors cannot happen in practice, because those events are counted at most once per clock cycle, and the number of clock cycles in a single execution is bounded by the maximal continuous operating time of the system.

We therefore designed a parametric abstract domain. (In our case, the parameter is the interval domain [5].) Let $X^{\sharp}$ be an abstract domain for a single scalar variable. The elements of the clocked domain consist in triples in $\left(X^{\sharp}\right)^{3}$. A triple $\left(v^{\sharp}, v_{-}^{\sharp}, v_{+}^{\sharp}\right)$ represents the set of values $x$ such that $x \in \gamma\left(v^{\sharp}\right), x-$ clock $\in \gamma\left(v_{-}^{\sharp}\right)$ and $x+$ clock $\in \gamma\left(v_{+}^{\sharp}\right)$, where clock is a special, hidden variable incremented each time the analyzed program waits for the next clock signal.

\subsubsection{The Octagon Abstract Domain}

Consider the following program fragment:

$$
\begin{aligned}
& \mathrm{R}:=\mathrm{X}-\mathrm{Z} \\
& \mathrm{L}:=\mathrm{X} \\
& \text { if }(\mathrm{R}>\mathrm{V}) \quad \mathrm{L}:=\mathrm{Z}+\mathrm{V} ;
\end{aligned}
$$

At the end of this fragment, we have $\mathrm{L} \leq \mathrm{X}$. In order to prove this, the analyzer must discover that, when the test is true, we have $\mathrm{R}=\mathrm{X}-\mathrm{Z}$ and $\mathrm{R}>\mathrm{V}$, and deduce from this that $\mathrm{Z}+\mathrm{V}<\mathrm{X}$ (up to rounding). This is possible only with a relational domain able to capture simple linear inequalities between variables.

Several such domains have been proposed, such as the widespread polyhedron domain [13]. In our prototype, we have chosen the recently developed octagon abstract domain $[28,30]$, which is less precise but faster than the polyhedron domain: it can represent sets of constraints of the form $\pm x \pm y \leq c$, and its complexity is cubic in time and quadratic in space (w.r.t. the number of variables), instead of exponential for polyhedra. Even with this reduced cost, the huge number of live variables prevents us from representing sets of concrete environments as one big abstract state (as it was done for polyhedra in [13]). Therefore we partition the set of variables into small subsets and use one octagon for some of these subsets (such a group of variables being then called a pack). The set of packs is a parameter of the analysis which can be determined automatically (Sect. 7.2.1).

Another reason for choosing octagons is the lack of support for floating-point arithmetics in the polyhedron domain. Designing relational domains for floating-point variables is indeed a difficult task, not much studied until recently [27]. On one hand, the abstract domain must be sound with respect to the concrete floating-point semantics (handling rounding, $\mathrm{NaNs}$, etc.); on the other hand it should use floating-point numbers internally to manipulate abstract data for the sake of efficiency. Because invariant manipulations in relational domains rely on some properties of the real field not true for floating-points (such as $x+y \leq c$ and 
$z-y \leq d$ implies $x+z \leq c+d)$, it is natural to consider that abstract values represent subsets of $\mathbb{R}^{N}$ (in the relational invariant $x+y \leq c$, the addition + is considered in $\mathbb{R}$, without rounding, overflow, etc.). Our solution separates the problem in two. First, we design a sound abstract domain for variables in the real field (our prototype uses the octagon library [28] which implementation is described in [29]). This is much easier for octagons than for polyhedra, as most computations are simple (addition, multiplication and division by 2). Then, each floating-point expression is transformed into a sound approximate real expression taking rounding, overflow, etc. into account (we use the linear forms described in Sect. 6.3) and evaluated by the abstract domain.

Coming back to our example, it may seem that octagons are not expressive enough to find the correct invariant as $\mathrm{Z}+\mathrm{V}<\mathrm{X}$ is not representable in an octagon. However, our assignment transfer function is smart enough to extract from the environment the interval $[c, d]$ where $\mathrm{V}$ ranges (with $d \leq \mathrm{R}_{M}$ where $\mathrm{R}_{M}$ is an upper bound of $\mathrm{R}$ already computed by the analysis) and synthesize the invariant $c \leq \mathrm{L}-\mathrm{Z} \leq d$, which is sufficient to prove that subsequent operations on $\mathrm{L}$ will not overflow. Thus, there was no need for this family of programs to use a more expressive and costly relational domain.

Remark that this approach provides a generic way of implementing relational abstract domains on floating-point numbers. It is parametrized by:

- a strategy for the determination of packs (Sect. 7.2.1);

- an underlying abstract domain working in the real field. Aspects specific to floating-point computation (such as rounding and illegal operations) are automatically taken care of by our approach.

\subsubsection{The Ellipsoid Abstract Domain}

To achieve the necessary precision, several new abstract domains had to be designed. We illustrate the general approach on the case of the ellipsoid abstract domain.

By inspection of the parts of the program on which the previously described analyses provide no information at all on the values of some program variables, we identified code of the form:

$$
\begin{aligned}
& \text { if }(\mathrm{B})\{ \\
& \mathrm{Y}:=i \\
& \mathrm{X}:=j \\
& \} \text { else }\{ \\
& \mathrm{X}^{\prime}:=a \mathrm{X}-b \mathrm{Y}+t \\
& \mathrm{Y}:=\mathrm{X} \\
& \mathrm{X}:=\mathrm{X}^{\prime}
\end{aligned}
$$

where $a$ and $b$ are floating-point constants, $i, j$ and $t$ are floating-point expressions, $\mathrm{B}$ is a boolean expression, and $\mathrm{X}$, $\mathrm{X}^{\prime}$, and $\mathrm{Y}$ are program variables. The previously described analyses yield the imprecise result that $\mathrm{X}$ and $\mathrm{Y}$ may take any value. This apparently specialized style of code is indeed quite frequent in control systems since it implements the simplified second order digital filtering discrete-time system illustrated in Fig. 1.

The first branch is a reinitialization step, the second branch consists in an affine transformation $\Phi$. Since this code is repeated inside loops, the analysis has to find an invariant preserved by this code. We looked manually for such an invariant on typical examples, identified the above

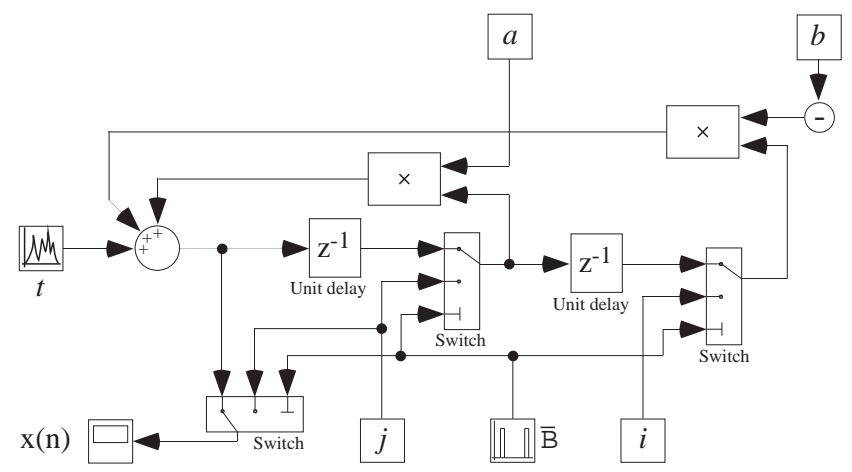

Figure 1: A simplified second-order digital filtering system.

generic form (essentially depending on $a$ and $b$ ), then designed a generic abstract domain $\varepsilon_{a, b}$ able to discover such invariants, implemented the abstract domain lattice and transfer operations and finally let the analyzer automatically instantiate the specific analysis to the code (in particular to parts that may not have been inspected).

To find an interval that contains the values of $\mathrm{X}$ and $\mathrm{Y}$ in the specific case where we can compute bounds to the expression $t$ by the previously described analyses, say $|t| \leq$ $t_{M}$, we have designed a new abstract domain $\varepsilon_{a, b}$ based on ellipsoids, that can capture the required invariant. More precisely, we can show that:

Proposition 1 If $0<b<1, a^{2}-4 b<0$, and $k \geq$ $\left(\frac{t_{M}}{1-\sqrt{b}}\right)^{2}$, then the constraint $\mathrm{X}^{2}-a \mathrm{XY}+b \mathrm{Y}^{2} \leq k$ is preserved by the affine transformation $\Phi$.

The proof of this proposition follows by algebraic manipulations using standard linear algebra techniques. In our examples, the conditions on $a$ and $b$ required in Prop. 1 are satisfied. We still have to design the abstract operations to propagate the invariant in the program, and to take into account rounding errors that occur in floating-point computations (and are not modeled in the above proposition).

Having fixed two floating-point numbers $a$ and $b$ such that $0<b<1$ and $a^{2}-4 b<0$, we present a domain $\varepsilon_{a, b}$, for describing sets of ellipsoidal constraints. An element in $\varepsilon_{a, b}$ is a function $r$ which maps a pair of variables $(\mathrm{X}, \mathrm{Y})$ to a floating-point number $r(\mathrm{X}, \mathrm{Y})$ such that $\mathrm{X}^{2}-a \mathrm{XY}+b \mathrm{Y}^{2} \leq$ $r(\mathrm{X}, \mathrm{Y})$.

We briefly describe some primitives and transfer functions of our domain:

- Assignments. Let $r \in \varepsilon_{a, b}$ be the abstract element describing some constraints before a statement $\mathrm{X}:=e$, our goal is to compute the abstract element $r^{\prime}$ describing a set of constraints satisfied after this statement:

1. in case $e$ is a variable $\mathrm{Y}$, each constraint containing $\mathrm{Y}$ gives a constraint for X. Formally, we take $r^{\prime}$ such that $r^{\prime}(U, V)=r(\sigma U, \sigma V)$ where $\sigma$ is the substitution of the variable $\mathrm{Y}$ for the variable $\mathrm{X}$;

2. in case $e$ is an expression of the form $a \mathrm{Y}+b \mathrm{Z}+t$, we first remove any constraint containing $\mathrm{X}$, then we add a new constraint for $\mathrm{X}$ and $\mathrm{Y}$. We therefore take:

$$
r^{\prime}=r\left[\left(\mathrm{X},{ }_{-}\right) \mapsto+\infty\right][(-, \mathrm{X}) \mapsto+\infty][(\mathrm{X}, \mathrm{Y}) \mapsto \delta(r(\mathrm{Y}, \mathrm{Z}))] .
$$


We have used the function $\delta$ defined as follows:

$$
\delta(k)=\left(\left(\sqrt{b}+\left(4 f \frac{|a| \sqrt{b}+b}{\sqrt{4 b-a^{2}}}\right)\right) \sqrt{k}+(1+f) t_{M}\right)^{2}
$$

where $f$ is the greatest relative error of a float with respect to a real and $t \in\left[-t_{M}, t_{M}\right]$. Indeed, we can show that, if $\mathrm{Y}^{2}-a \mathrm{YZ}+b \mathrm{Z}^{2} \leq k$ and $\mathrm{X}=a \mathrm{Y}-b \mathrm{Z}+t$, then in exact real arithmetic $\mathrm{X}^{2}-a \mathrm{XY}+b \mathrm{Y}^{2} \leq\left(\sqrt{b k}+t_{M}\right)^{2}$, and taking into account rounding errors, we get the above formula for $\delta(k)$;

3. otherwise, we remove all constraints containing $\mathrm{X}$ by taking $r^{\prime}=r\left[\left(\mathrm{X},{ }_{-}\right) \mapsto+\infty\right][(-, \mathrm{X}) \mapsto+\infty]^{3}$.

- Guards are ignored, i.e., $r^{\prime}=r$.

- Abstract union, intersection, widening and narrowing are computed component-wise. The widening uses thresholds as described in Sect. 7.1.2.

The abstract domain $\varepsilon_{a, b}$ cannot compute accurate results by itself, mainly because of inaccurate assignments (in case 3.) and guards. Hence we use an approximate reduced product with the interval domain. A reduction step consists in substituting in the function $r$ the image of a couple $(\mathrm{X}, \mathrm{Y})$ by the smallest element among $r(\mathrm{X}, \mathrm{Y})$ and the floatingpoint number $k$ such that $k$ is the least upper bound to the evaluation of the expression $\mathrm{X}^{2}-a \mathrm{XY}+b \mathrm{Y}^{2}$ in the floatingpoint numbers when considering the computed interval constraints. In case the values of the variable $\mathrm{X}$ and $\mathrm{Y}$ are proved to be equal, we can be much more precise and take the smallest element among $r(\mathrm{X}, \mathrm{Y})$ and the least upper bound to the evaluation of the expression $(1-a+b) \mathrm{X}^{2}$.

These reduction steps are performed:

- before computing the union between two abstract elements $r_{1}$ and $r_{2}$, we reduce each constraint $r_{i}(\mathrm{X}, \mathrm{Y})$ such that $r_{i}(\mathrm{X}, \mathrm{Y})=+\infty$ and $r_{3-i}(\mathrm{X}, \mathrm{Y}) \neq+\infty$ (where $\left.i \in\{1 ; 2\}\right)$;

- before computing the widening between two abstract elements $r_{1}$ and $r_{2}$, we reduce each constraint $r_{2}(\mathrm{X}, \mathrm{Y})$ such that $r_{2}(\mathrm{X}, \mathrm{Y})=+\infty$ and $r_{1}(\mathrm{X}, \mathrm{Y}) \neq+\infty$;

- before an assignment of the form $\mathrm{X}^{\prime}:=a \mathrm{X}-b \mathrm{Y}+t$, we refine the constraints $r(\mathrm{X}, \mathrm{Y})$.

These reduction steps are especially useful in handling a reinitialization iteration.

Ellipsoidal constraints are then used to reduce the intervals of variables: after each assignment $A$ of the form $\mathrm{X}^{\prime}:=a \mathrm{X}-b \mathrm{Y}+t$, we use the fact that $\left|\mathrm{X}^{\prime}\right| \leq 2 \sqrt{b} \sqrt{\frac{r^{\prime}\left(\mathrm{X}^{\prime}, \mathrm{X}\right)}{4 b-a^{2}}}$, where $r^{\prime}$ is the abstract element describing a set of ellipsoidal constraints just after the assignment $A$.

This approach is generic and has been applied to handle the digital filters in the program.

\subsubsection{The Decision Tree Abstract Domain}

Apart from numerical variables, the code uses also a great deal of boolean values, and no classical numerical domain deals precisely enough with booleans. In particular, booleans can be used in the control flow and we need to relate the value of the booleans to some numerical variables. Here is an example:

$$
\begin{aligned}
& \mathrm{B}:=(\mathrm{X}=0) ; \\
& \text { if }(\neg \mathrm{B}) \quad \mathrm{Y}:=1 / \mathrm{X} ;
\end{aligned}
$$

We found also more complex examples where a numerical variable could depend on whether a boolean value had

\footnotetext{
3 This is also the case for initialization.
}

changed or not. In order to deal precisely with those examples, we implemented a simple relational domain consisting in a decision tree with leaf an arithmetic abstract domain ${ }^{4}$. The decision trees are reduced by ordering boolean variables (as in [6]) and by performing some opportunistic sharing of subtrees.

The only problem with this approach is that the size of decision trees can be exponential in the number of boolean variables, and the code contains thousands of global ones. So we extracted a set of variable packs, and related the variables in the packs only, as explained in Sect. 7.2.3.

\subsection{Symbolic Manipulation of Expressions}

We observed, in particular for non-relational abstract domains, that transfer functions proceeding by structural induction on expressions are not precise when the variables in the expression are not independent. Consider, for instance, the simple assignment $\mathrm{X}:=\mathrm{X}-0.2 * \mathrm{X}$ performed in the interval domain in the environment $X \in[0,1]$. Bottomup evaluation will give $\mathrm{X}-0.2 * \mathrm{X} \Rightarrow[0,1]-0.2 *[0,1] \Rightarrow$ $[0,1]-[0,0.2] \Rightarrow[-0.2,1]$. However, because the same $\mathrm{X}$ is used on both sides of the - operator, the precise result should have been $[0,0.8]$.

In order to solve this problem, we perform some simple algebraic simplifications on expressions before feeding them to the abstract domain. Our approach is to linearize each expression e, that is to say, transform it into a linear form $\ell \llbracket \mathbf{e} \rrbracket$ on the set of variables $v_{1}, \ldots, v_{N}$ with interval coefficients: $\ell \llbracket \mathbf{e} \rrbracket=\sum_{i=1}^{N}\left[\alpha_{i}, \beta_{i}\right] v_{i}+[\alpha, \beta]$. The linear form $\ell \llbracket \mathbf{e} \rrbracket$ is computed by recurrence on the structure of $\mathbf{e}$. Linear operators on linear forms (addition, subtraction, multiplication and division by a constant interval) are straightforward. For instance, $\ell \llbracket \mathrm{X}-0.2 * \mathrm{X} \rrbracket=0.8 * \mathrm{X}$, which will be evaluated to $[0,0.8]$ in the interval domain. Non-linear operators (multiplication of two linear forms, division by a linear form, nonarithmetic operators) are dealt by evaluating one or both linear form argument into an interval.

Although the above symbolic manipulation is correct in the real field, it does not match the semantics of $\mathrm{C}$ expressions for two reasons:

- floating-point computations incur rounding;

- errors (division by zero, overflow, etc.) may occur.

Thankfully, the systems we consider conform to the IEEE 754 norm [23] that describes rounding very well (so that, e.g., the compiler should be prevent from using the multiplyadd-fused instruction on machines for which the result of a multiply-add computation may be slightly different from the floating point operation operation $A+(B \times C)$ for some input values $A, B, C)$. Thus, it is easy to modify the recursive construction of linear forms from expressions to add the error contribution for each operator. It can be an absolute error interval, or a relative error expressed as a linear form. We have chosen the absolute error which is more easily implemented and turned out to be precise enough.

To address the second problem, we first evaluate the expression in the abstract interval domain and proceed with the linearization to refine the result only if no possible arithmetic error was reported. We are then guaranteed that the simplified linear form has the same semantics as the initial expression.

\footnotetext{
${ }^{4}$ The arithmetic abstract domain is generic. In practice, the interval domain was sufficient.
} 


\section{ADAPTATION VIA PARAMETRIZA- TION}

In order to adapt the analyzer to a particular program of the considered family, it may be necessary to provide information to help the analysis. A classical idea is to have users provide assertions (which can be proved to be invariants and therefore ultimately suppressed). Another idea is to use parametrized abstract domains in the static program analyzer. Then the static analysis can be adapted to a particular program by an appropriate choice of the parameters. We provide several examples in this section. Moreover we show how the analyzer itself can be used in order to help or even automatize the appropriate choice of these parameters.

\subsection{Parametrized Iteration Strategies}

\subsubsection{Loop Unrolling}

In many cases, the analysis of loops is made more precise by treating the first iteration of the loop separately from the following ones; this is simply a semantic loop unrolling transformation: a while loop may be expanded as follows:

$$
\text { if (condition) \{ body; while (condition) }\{\text { body }\}\}
$$

The above transformation can be iterated $n$ times, where the concerned loops and the unrolling factor $n$ are user-defined parameters. In general, the larger the $n$, the more precise the analysis, and the longer the analysis time.

\subsubsection{Widening with Thresholds}

Compared to normal interval analysis [10, §2.1.2], ours does not jump straight away to $\pm \infty$, but goes through a number of thresholds. The widening with thresholds $\nabla_{T}$ for the interval analysis of Sect. 6.2.1 is parametrized by a threshold set $T$ that is a finite set of numbers containing $-\infty$ and $+\infty$ and defined such that:

$$
\begin{array}{r}
{[a, b] \nabla_{T}\left[a^{\prime}, b^{\prime}\right]=\left[\text { if } a^{\prime}<a \text { then } \max \left\{\ell \in T \mid \ell \leq a^{\prime}\right\} \text { else } a,\right.} \\
\text { if } \left.b^{\prime}>b \text { then } \min \left\{h \in T \mid h \geq b^{\prime}\right\} \text { else } b\right]
\end{array}
$$

In order to illustrate the benefits of this parametrization (see others in [5]), let $x_{0}$ be the initial value of a variable $\mathrm{X}$ subject to assignments of the form $\mathrm{X}:=\alpha_{i} * \mathrm{X}+\beta_{i}, i \in \Delta$ in the main loop, where the $\alpha_{i}, \beta_{i}, i \in \Delta$ are floating point constants such that $0 \leq \alpha_{i}<1$. Let be any $M$ such that $M \geq \max \left\{\left|x_{0}\right|, \frac{\left|\beta_{i}\right|}{1-\alpha_{i}}, i \in \Delta\right\}$. We have $M \geq\left|x_{0}\right|$ and $M \geq \alpha_{i} M+\left|\beta_{i}\right|$ and so all possible sequences $x^{0}=x_{0}$, $x^{n+\overline{1}}=\alpha_{i} x^{n}+\beta_{i}, i \in \Delta$ of values of variable $\mathrm{X}$ are bounded since $\forall n \geq 0:\left|x^{n}\right| \leq M$. Discovering $M$ may be difficult in particular if the constants $\alpha_{i}, \beta_{i}, i \in \Delta$ depend on complex boolean conditions. As long as the set $T$ of thresholds contains some number greater or equal to the minimum $M$, the interval analysis of $\mathrm{X}$ with thresholds $T$ will prove that the value of $\mathrm{X}$ is bounded at run-time since some element of $T$ will be an admissible $M$.

In practice we have chosen $T$ to be $\left( \pm \alpha \lambda^{k}\right)_{0 \leq k \leq N}$. The choice of $\alpha$ and $\lambda$ mostly did not matter much in the first experiments. After the analysis had been well refined and many causes of imprecision removed, we had to choose a smaller value for $\lambda$ to remove some false alarms. In any case, $\alpha \lambda^{N}$ should be large enough; otherwise, many false alarms for overflow are produced.

\subsubsection{Delayed Widening}

When widening the previous iterate by the result of the transfer function on that iterate at each step as in Sect. 5.5, some values which can become stable after two steps of widening may not stabilize. Consider the example:

$$
\begin{aligned}
& \mathrm{X}:=\mathrm{Y}+\gamma ; \\
& \mathrm{Y}:=\alpha * \mathrm{X}+\delta
\end{aligned}
$$

This should be equivalent to $\mathrm{Y}:=\alpha * \mathrm{Y}+\beta$ (with $\beta=$ $\delta+\alpha \gamma$ ), and so a widening with thresholds should find a stable interval. But if we perform a widening with thresholds at each step, each time we widen $Y, X$ is increased to a value surpassing the threshold for $\mathrm{Y}$, and so $\mathrm{X}$ is widened to the next stage, which in turn increases $Y$ further and the next widening stage increases the value of $Y$. This eventually results in top abstract values for $\mathrm{X}$ and $\mathrm{Y}$.

In practice, we first do $N_{0}$ iterations with unions on all abstract domains, then we do widenings unless a variable which was not stable becomes stable (this is the case of $\mathrm{Y}$ here when the threshold is big enough as described in Sect. 7.1.2). We add a fairness condition to avoid livelocks in cases for each iteration there exists a variable that becomes stable.

\subsubsection{Floating Iteration Perturbation}

The stabilization check for loops considered in Sect. 5.4 has to be adjusted because of the floating point computations in the abstract. Let us consider that $[a, b]$ is the mathematical interval of values of a variable $\mathrm{X}$ on entry of a loop. We let $F_{\mathbb{C}, \mathbb{A}}([a, b])$ be the mathematical interval of values of $\mathrm{X}$ after a loop iteration. $\mathbb{C}=\mathbb{R}$ means that the concrete operations in the loop are considered to be on mathematical real numbers while $\mathbb{C}=\mathbb{F}$ means that the concrete operations in the loop are considered to be on machine floating point numbers. If $F_{\mathbb{R}, \mathbb{A}}([a, b])=\left[a^{\prime}, b^{\prime}\right]$ then $F_{\mathbb{F}, \mathbb{A}}([a, b])=$ $\left[a^{\prime}-\epsilon_{1}, b^{\prime}+\epsilon_{1}\right]$ because of the cumulated concrete rounding errors $\epsilon_{1} \geq 0$ when evaluating the loop body ${ }^{5}$. The same way $\mathbb{A}=\mathbb{R}$ means that the interval abstract domain is defined ideally using mathematical real numbers while $\mathbb{A}=\mathbb{F}$ means that the interval abstract domain is implemented with floating point operations performing rounding in the right direction. Again, if $F_{\mathbb{C}, \mathbb{R}}([a, b])=\left[a^{\prime}, b^{\prime}\right]$ then $F_{\mathbb{C}, \mathbb{F}}([a, b])=\left[a^{\prime}-\epsilon_{2}, b^{\prime}+\epsilon_{2}\right]$ because of the cumulated abstract rounding errors during the static analysis of the loop body. The analyzer might use $F_{\mathbb{F}, \mathbb{F}}$ which is sound since if $F_{\mathbb{R}, \mathbb{R}}([a, b])=\left[a^{\prime}, b^{\prime}\right]$ then $F_{\mathbb{F}, \mathbb{F}}([a, b])=\left[a^{\prime}-\epsilon_{1}-\epsilon_{2}, b^{\prime}+\epsilon_{1}+\epsilon_{2}\right]$ which takes both the concrete and abstract rounding errors into account (respectively $\epsilon_{1}$ and $\epsilon_{2}$ ).

Mathematically, a loop invariant for variable $\mathrm{X}$ is an interval $[a, b]$ such that $F_{\mathbb{F}, \mathbb{R}}([a, b]) \subseteq[a, b]$. However, the loop stabilization check is made as $F_{\mathbb{F}, \mathbb{F}}([a, b]) \subseteq[a, b]$, which is sound but incomplete: if $F_{\mathbb{F}, \mathbb{R}}([a, b])$ is very close to $[a, b]$, e.g. $F_{\mathbb{F}, \mathbb{R}}([a, b])=[a, b]$ then, unfortunately, $F_{\mathbb{F}, \mathbb{F}}([a, b])=$ $\left[a-\epsilon_{2}, b+\epsilon_{2}\right] \nsubseteq[a, b]$. This will launch useless additional iterations whence a loss of time and precision.

The solution we have chosen is to overapproximate $F_{\mathbb{F}, \mathbb{F}}$ by $\widehat{F}_{\mathbb{F}, \mathbb{F}}$ such that $\widehat{F}_{\mathbb{F}, \mathbb{F}}([a, b])=\left[a^{\prime}-\epsilon *\left|a^{\prime}\right|, b^{\prime}+\epsilon *\left|b^{\prime}\right|\right]$ where $\left[a^{\prime}, b^{\prime}\right]=F_{\mathbb{F}, \mathbb{F}}([a, b])$ and $\epsilon$ is a parameter of the analyzer chosen to be an upper bound of the possible abstract rounding errors in the program loops. Then the loop invariant inter-

\footnotetext{
${ }^{5}$ We take the rounding error on the lower and upper bound to be the same for simplicity.
} 
val is computed iteratively with $\widehat{F}_{\mathbb{F}, \mathbb{F}}$, which is simply less precise than with $F_{\mathbb{F}, \mathbb{F}}$, but sound. The loop stabilization test is performed with $F_{\mathbb{F}, \mathbb{F}}$ which is sound. It is also more precise in case $\epsilon *\left(\min \left\{\left|a^{\prime}\right| ;\left|b^{\prime}\right|\right\}\right)$ is greater than the absolute error on the computation of $F_{\mathbb{F}, \mathbb{F}}\left(\left[a^{\prime}-\epsilon *\left|a^{\prime}\right|, b^{\prime}+\epsilon *\left|b^{\prime}\right|\right]\right)$. We have not investigated about the existence (nor about the automatic computation) of such a parameter in the general case yet, nevertheless attractiveness of the encountered fixpoints made the chosen parameter convenient.

\subsubsection{Trace Partitioning}

In the abstract execution of the program, when a test is met, both branches are executed and then the abstract environments computed by each branch are merged. As described in [5] we can get a more precise analysis by delaying this merging.

This means that:

$$
\text { if }(c)\left\{S_{1}\right\} \text { else }\left\{S_{2}\right\} \text { rest }
$$

is analyzed as if it were

$$
\text { if }(c)\left\{S_{1} \text {; rest }\right\} \text { else }\left\{S_{2} ; \text { rest }\right\} \text {. }
$$

A similar technique holds for the unrolled iterations of loops. As this process is quite costly, the analyzer performs this trace partitioning in a few end-user selected functions, and the traces are merged at the return point of the function. Informally, in our case, the functions that need partitioning are those iterating simultaneously over arrays $a[]$ and $b[]$ such that $\mathrm{a}[i]$ and $\mathrm{b}[i]$ are linked by an important numerical constraint which does not hold in general for a $[i]$ and $\mathrm{b}[j]$ where $i \neq j$. This solution was simpler than adding complex invariants to the abstract domain.

\subsection{Parametrized Abstract Domains}

Recall that our relational domains (octagons of Sect. 6.2.2, and decision trees of Sect. 6.2.4) operate on small packs of variables for efficiency reasons. This packing is determined syntactically before the analysis. The packing strategy is a parameter of the analysis; it gives a trade-off between accuracy (more, bigger packs) and speed (fewer, smaller packs). The strategy must also be adapted to the family of programs to be analyzed.

\subsubsection{Packing for Octagons}

We determine a set of packs of variables and use one octagon for each pack. Packs are determined once and for all, before the analysis starts, by examining variables that interact in linear assignments within small syntactic blocks (curly-brace delimited blocks). One variable may appear in several packs and we could do some information propagation (i.e. reduction [9]) between octagons at analysis time, using common variables as pivots; however, this precision gain was not needed in our experiments. There is a great number of packs, but each pack is small; it is our guess that our packing strategy constructs, for our program family, a linear number of constant-sized octagons, effectively resulting in a cost linear in the size of the program. Moreover, the octagon packs are efficiently manipulated using functional maps, as explained in Sect. 6.1.2, to achieve sub-linear time costs via sharing of unmodified octagons.

Our current strategy is to create one pack for each syntactic block in the source code and put in the pack all variables that appear in a linear assignment or test within the associated block, ignoring what happens in sub-blocks of the block. For example, on a program of $75 \mathrm{kLOC}, 2,600$ octagons were detected, each containing four variables on average. Larger packs (resulting in increased cost and precision) could be created by considering variables appearing in one or more levels of nested blocks; however, we found that, in our program family, it does not improve precision.

\subsubsection{Packing Optimization for Octagons}

Our analyzer outputs, as part of the result, whether each octagon actually improved the precision of the analysis. It is then possible to re-run the analysis using only packs that were proven useful, thus greatly reducing the cost of the analysis. (In our $75 \mathrm{kLOC}$ example, only 400 out of the 2,600 original octagons were in fact useful.) Even when the program or the analysis parameters are modified, it is perfectly safe to use a list of useful packs output by a previous analysis. We experimented successfully with the following method: generate at night an up-to-date list of good octagons by a full, lengthy analysis and work the following day using this list to cut analysis costs.

\subsubsection{Packing for Decision Trees}

In order to determine useful packs for the decision trees of Sect. 6.2.4, we used the following strategy: each time a numerical variable assignment depends on a boolean, or a boolean assignment depends on a numerical variable, we put both variables in a tentative pack. If, later, we find a program point where the numerical variable is inside a branch depending on the boolean, we mark the pack as confirmed. In order to deal with complex boolean dependences, if we find an assignment $\mathrm{b}:=\operatorname{expr}$ where expr is a boolean expression, we add $\mathrm{b}$ to all packs containing a variable in expr. In the end, we just keep the confirmed packs.

At first, we restrained the boolean expressions used to extend the packs to simple boolean variables (we just considered $\mathrm{b}:=\mathrm{b}^{\prime}$ ) and the packs contained at most four boolean variables and dozens of false alarms were removed. But we discovered that more false alarms could be removed if we extended those assignments to more general expressions. The problem was that packs could then contain up to 36 boolean variables, which gave very bad performance. So we added a parameter to restrict arbitrarily the number of boolean variables in a pack. Setting this parameter to three yields an efficient and precise analysis of boolean behavior.

\section{EXPERIMENTAL RESULTS}

The main program we are interested in is 132,000 lines of $\mathrm{C}$ with macros $(75 \mathrm{kLOC}$ after preprocessing and simplification as in Sect. 5.1) and has about 10,000 global/static variables (over 21,000 after array expansion as in Sect. 6.1). We had 1,200 false alarms with the analyzer [5] we started with. The refinements of the analyzer described in this paper reduce the number of alarms down to 11 (and even 3, depending on the versions of the analyzed program). Fig. 2 gives the total analysis time for a family of related programs on commodity hardware (2.4 GHz, 1 Gb RAM PC), using a slow but precise iteration strategy.

The memory consumption of the analyzer is reasonable (550 Mb for the full-sized program). Several parameters, for instance the size of the octagon packs (Sect. 7.2.1), allow for a space-precision trade-off.

The packing optimization strategy of reusing results from preceding analysis to reduce the number of octagons 


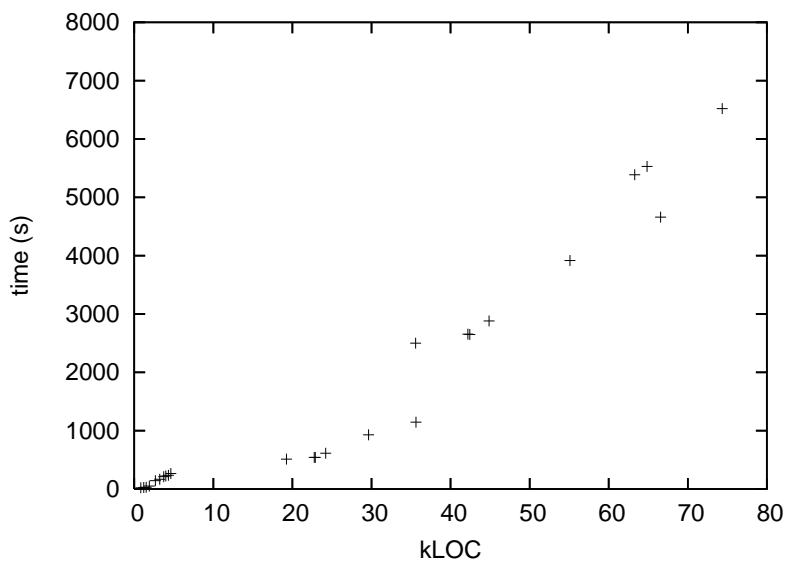

Figure 2: Total analysis time for the family of programs without packing optimization (Sect. 7.2.2).

(Sect. 7.2.2) reduces, on the largest example code, memory consumption from $550 \mathrm{Mb}$ to $150 \mathrm{Mb}$ and time from $1 \mathrm{~h} 40 \mathrm{~min}$ to $40 \mathrm{~min}$. Furthermore, the current automatic tuning of the iteration strategy may be made more efficient, using fewer iterations and thus reducing analysis time.

\section{RELATED WORK}

Let us discuss some other verification methods that could have been considered. Dynamic checking methods were excluded for a safety critical system (at best data can be collected at runtime and checked offline). Static methods requiring compiler or code instrumentation (such as [15]) were also excluded in our experiment since the certified compiler as well as the compiled code, once certified by traditional methods, cannot be modified without costly re-certification processes. Therefore we only consider the automated static proof of software run-time properties, which has been a recurrent research subject since a few decades.

\subsection{Software Model Checking}

Software model checking [22] has proved very useful to trace logical design errors, which in our case has already been performed at earlier stages of the software development, whereas we concentrate on abstruse machine implementation aspects of the software. Building a faithful model of the program (e.g. in PROMELA for SPIN [22]) would be just too hard (it can take significantly more time to write a model than it did to write the code) and error-prone (by checking a manual abstraction of the code rather than the code itself, it is easy to miss errors). Moreover the abstract model would have to be designed with a finite state space small enough to be fully explored (in the context of verification, not just debugging), which is very difficult in our case since sharp data properties must be taken into account. So it seems important to have the abstract model automatically generated by the verification process, which is the case of the abstract semantics in static analyzers.

\subsection{Dataflow Analysis and Software Abstract Model Checking}

Dataflow analyzers (such as ESP [14]) as well as abstraction based software model checkers (such as a.o. BLAST [21], CMC [31] and SLAM [4]) have made large inroads in tackling programs of comparable size and complexity. Their impressive performance is obtained thanks to coarse abstractions (e.g. resulting from a program "shrinking" preprocessing phase $[14,1]$ or obtained by a globally coarse but locally precise abstraction [31]). In certain cases, the abstract model is just a finite automaton, whose transitions are triggered by certain constructions in the source code [15]; this allow checking at the source code level high-level properties, such as "allocated blocks of memory are freed only once" or "interrupts are always unmasked after being blocked", ignoring dependencies on data.

The benefit of this coarse abstraction is that only a small part of the program control and/or data have to be considered in the actual verification process. This idea did not work out in our experiment since merging paths or data inevitably leads to many false alarms. On the contrary we had to resort to context-sensitive polyvariant analyses (Sect. 5.4) with loop unrolling (Sect. 7.1.1) so that the size of the (semantically) "expanded" code to analyze is much larger than that of the original code. Furthermore, the properties we prove include fine numerical constraints, which excludes simple abstract models.

\subsection{Deductive Methods}

Proof assistants (such as CoQ [33], ESC [17] or PVS [32]) face semantic problems when dealing with real-life programming languages. First, the prover has to take the machinelevel semantics into account (e.g., floating-point arithmetic with rounding errors as opposed to real numbers, which is far from being routinely available ${ }^{6}$ ). Obviously, any technique for analyzing machine arithmetic will face the same semantic problems. However, if the task of taking concrete and rounding errors into account turned out to be feasible for our automated analyzer, this task is likely to be daunting in the case of complex decision procedures operating on ideal arithmetic [32]. Furthermore, exposing to the user the complexity brought by those errors is likely to make assisted manual proof harrowing.

A second semantic difficulty is that the prover needs to operate on the $\mathrm{C}$ source code, not on some model written in a prototyping language so that the concrete program semantics must be incorporated in the prover (at least in the verification condition generator). Theoretically, it is possible to do a "deep embedding" of the analyzed program into the logic of the proof assistant - that is, providing a mathematical object describing the syntactic structure of the program as well as a formal semantics of the programming language. Proving any interesting property is then likely to be extremely difficult. "Shallow embeddings" - mapping the original program to a corresponding "program" in the input syntax of the prover - are easier to deal with, but may be difficult to produce in the presence of nondeterministic inputs, floating-point rounding errors etc...

The last and main difficulty with proof assistants is that they must be assisted, in particular to help providing inductive arguments (e.g. invariants). Of course these provers could integrate abstract domains in the form of abstraction procedures (to perform online abstractions of arbitrary predicates into their abstract form) as well as decision procedures (e.g. to check for abstract inclusion $\sqsubseteq^{\sharp}$ ). The main problem is to have the user provide program independent

\footnotetext{
${ }^{6}$ For example ESC is simply unsound with respect to modular arithmetics [17].
} 
hints, specifying when and where these abstraction and decision procedures must be applied, as well as how the inductive arguments can be discovered, e.g. by iterative fixpoint approximation, without ultimately amounting to the implementation of a static program analysis.

Additionally, our analyzer is designed to run on a whole family of software, requiring minimal adaptation to each individual program. In most proof assistants, it is difficult to change the program without having to do a considerable amount of work to adapt proofs.

\subsection{Predicate Abstraction}

Predicate abstraction, which consists in specifying an abstraction by providing the atomic elements of the abstract domain in logical form [19] e.g. by representing sets of states as boolean formulas over a set of base predicates, would certainly have been the best candidate. Moreover most implementations incorporate an automatic refinement process by success and failure [2, 21] whereas we successively refined our abstract domains manually, by experimentation. In addition to the semantic problems shared by proof assistants, a number of difficulties seem to be insurmountable to automate this design process in the present state of the art of deductive methods:

\subsubsection{State Explosion Problem:}

To get an idea of the size of the necessary state space, we have dumped the main loop invariant (a textual file over $4.5 \mathrm{Mb})$.

The main loop invariant includes 6,900 boolean interval assertions $(x \in[0,1]), 9,600$ interval assertions $(x \in[a, b])$, 25,400 clock assertions (Sect. 6.2.1), 19,100 additive octagonal assertions $(a \leq x+y \leq b), 19,200$ subtractive octagonal assertions ( $a \leq x-y \leq b$, see Sect. 6.2.2), 100 decision trees (Sect. 6.2.4) and 1,900 ellipsoidal assertions (Sect. 6.2.3) ${ }^{7}$.

In order to allow for the reuse of boolean model checkers, the conjunction of true atomic predicates is usually encoded as a boolean vector over boolean variables associated to each predicate [19] (the disjunctive completion [9] of this abstract domain can also be used to get more precision [2, 21 ], but this would introduce an extra exponential factor). Model checking state graphs corresponding to several tenths of thousands of boolean variables (not counting hundreds of thousands of program points) is still a real challenge. Moreover very simple static program analyzes, such as Kildall's constant propagation [24], involve an infinite abstract domain which cannot be encoded using finite boolean vectors thus requiring the user to provide beforehand all predicates that will be indispensable to the static analysis (for example the above mentioned loop invariant involves, e.g., over 16,000 floating point constants at most 550 of them appearing in the program text).

Obviously some of the atomic predicates automatically generated by our analysis might be superfluous. On one hand it is hard to say which ones and on the other hand this does not count all other predicates that may be indispensable at some program point to be locally precise. Another approach would consist in trying to verify each potential

\footnotetext{
${ }^{7}$ Figures are rounded to the closest hundred. We get more assertions than variables because in the 10,000 global variables arrays are counted once whereas the element-wise abstraction yields assertions on each array element. Boolean assertions are needed since booleans are integers in C.
}

faulty operation separately (e.g., focus on one instruction that may overflow at a time) and generate the abstractions lazily [21]. Even though repeating this analysis over 100,000 times might be tractable, the real difficulty is to automatically refine the abstract predicates (e.g. to discover that considered in Prop. 1).

\subsubsection{Predicate Refinement:}

Predicate abstraction per se uses a finite domain and is therefore provably less powerful than our use of infinite abstract domains (see [12], the intuition is that all inductive assertions have to be provided manually). Therefore predicate abstraction is often accompanied by a refinement process to cope with false alarms $[2,21]$.

Under specific conditions, this refinement can be proved equivalent to the use of an infinite abstract domain with widening [3].

Formally this refinement is a fixpoint computation $[7,18]$ at the concrete semantics level, whence introduces new elements in the abstract domain state by state without termination guarantee whereas, e.g., when introducing clocks from intervals or ellipsoids from octagons we exactly look for an opposite more synthetic point of view. Therefore the main difficulty of counterexample-based refinement is still to automate the presently purely intellectual process of designing precise and efficient abstract domains.

\section{CONCLUSION}

In this experiment, we had to cope with stringent requirements. Industrial constraints prevented us from requiring any change in the production chain of the code. For instance, it was impossible to suggest changes to the library functions that would offer the same functionality but would make the code easier to analyze. Furthermore, the code was mostly automatically generated from a high-level specification that we could not have access to, following rules of separation of design and verification meant to prevent the intrusion of unproved high-level assumptions into the verification assumptions. It was therefore impossible to analyze the high-level specification instead of analyzing the $\mathrm{C}$ code.

That the code was automatically generated had contrary effects. On the one hand, the code fit into some narrow subclass of the whole $\mathrm{C}$ language. On the other hand, it used some idioms not commonly found in human-generated code that may make the analysis more difficult; for instance, where a human would have written a single test with a boolean connective, the generated code would make one test, store the result into a boolean variable, do something else do the second test and then retrieve the result of the first test. Also, the code maintains a considerable number of state variables, a large number of these with local scope but unlimited lifetime. The interactions between several components are rather complex since the considered program implement complex feedback loops across many interacting components.

Despite those difficulties, we developed an analyzer with a very high precision rate, yet operating with reasonable computational power and time. Our main effort was to discover an appropriate abstraction which we did by manual refinement through experimentation of an existing analyzer [5] and can be later adapted by end-users to particular programs through parametrization (Sect. 6.3 and 7). To 
achieve this, we had to develop two specialized abstract domains (Sect. 6.2.3 and 6.2.4) and improve an existing domain (Sect. 6.2.2).

The central idea in this approach is that once the analyzer has been developed by specialists, end-users can adapt it to other programs in the family without much efforts. However coming up with a tool that is effective in the hands of end users with minimal expertise in program analysis is hard. This is why we have left to the user the simpler parametrizations only (such as widening thresholds in Sect. 7.1.2 easily found in the program documentation) and automated the more complex ones (such as parametrized packing Sect. 7.2). Therefore, the approach should be economically viable.

\section{REFERENCES}

[1] S. Adams, T. Ball, M. Das, S. Lerner, K. Rajamani, M. Seigle, , and W. Weimer. Speeding up dataflow analysis using flow-insensitive pointer analysis. $S A S$ (2002), LNCS 2477, Springer, 117-132.

[2] T. Ball, R. Majumdar, T. Millstein, and S. Rajamani. Automatic predicate abstraction of $\mathrm{C}$ programs. PLDI. ACM SIGPLAN Not. 36(5) (2001), 203-213.

[3] T. Ball, A. Podelski, and S. Rajamani. Relative completeness of abstraction refinement for software model checking. TACAS (2002), LNCS 2280, Springer, 158-172.

[4] T. Ball and S. Rajamani. The SLAM project: debugging system software via static analysis. $29^{\text {th }}$ ACM POPL (2002), 1-3.

[5] B. Blanchet, P. Cousot, R. Cousot, J. Feret, L. Mauborgne, A. Miné, D. Monniaux, and X. Rival. Design and implementation of a special-purpose static program analyzer for safety-critical real-time embedded software. The Essence of Computation: Complexity, Analysis, Transformation. (2002), LNCS 2566, Springer, 85-108.

[6] R. Bryant. Graph based algorithms for boolean function manipulation. IEEE Trans. Comput. C-35 (1986), 677-691.

[7] P. Cousot. Partial completeness of abstract fixpoint checking. SARA (2000), LNAI 1864, Springer, 1-25.

[8] P. Cousot and R. Cousot. Abstract interpretation: a unified lattice model for static analysis of programs by construction or approximation of fixpoints. $4^{\text {th }} A C M$ POPL (1977), 238-252.

[9] P. Cousot and R. Cousot. Systematic design of program analysis frameworks. $6^{\text {th }}$ ACM POPL (1979), 269-282.

[10] P. Cousot and R. Cousot. Abstract interpretation and application to logic programs. J. of Logic Prog. 2-3, 13 (1992), 103-179.

[11] P. Cousot and R. Cousot. Abstract interpretation frameworks. J. Logic and Comp. 2, 4 (1992), 511-547.

[12] P. Cousot and R. Cousot. Comparing the Galois connection and widening/narrowing approaches to abstract interpretation. PLILP (1992), LNCS 631, Springer, 269-295.

[13] P. Cousot and N. Halbwachs. Automatic discovery of linear restraints among variables of a program. $5^{\text {th }}$ ACM POPL (1978), 84-97.

[14] M. Das, S. Lerner, and M. Seigle. ESP: Path-sensitive program verification in polynomial time. $29^{\text {th }} A C M$
PLDI (2002), 58-70.

[15] D. Engler, B. Chelf, A. Chou, and S. Hallem. Checking system rules using system-specific, programmer-written compiler extensions. USENIX Association, OSDI 2000, 1-16.

[16] C. Ferdinand, R. Heckmann, M. Langenbach, F. Martin, M. Schmidt, H. Theiling, S. Thesing, and R. Wilhelm. Reliable and precise WCET determination for a real-life processor. ESOP (2001), LNCS 2211, Springer, 469-485.

[17] C. Flanagan, K. R. M. Leino, M. Lillibridge, G. Nelson, J. Saxe, and R. Stata. Extended static checking for Java. PLDI. ACM SIGPLAN Not. 37(5) (2002), 234-245.

[18] R. Giacobazzi and E. Quintarelli. Incompleteness, counterexamples and refinements in abstract model-checking. SAS (2001), LNCS 126, Springer, 356-373.

[19] S. Graf and H. Saïdi. Construction of abstract state graphs with PVS. CAV (1997), LNCS 1254, Springer, $72-83$.

[20] N. Halbwachs, P. Caspi, P. Raymond, and D. Pilaud. The synchronous dataflow programming language Lustre. Proc. of the IEEE 79, 9 (1991), 1305-1320.

[21] T. Henzinger, R. Jhala, R. Majumdar, and G. Sutre. Lazy abstraction. 29 $9^{\text {th }}$ ACM POPL (2002), 58-70.

[22] G. Holzmann. The model checker SpIn. IEEE Trans. Softw. Eng. 23, 5 (1997), 279-295.

[23] IEEE Computer Society. IEEE standard for binary floating-point arithmetic. Tech. rep., ANSI/IEEE Std 745-1985, 1985.

[24] G. Kildall. A unified approach to global program optimization. $1^{\text {st }}$ ACM POPL (1973.), 194-206.

[25] X. Leroy, D. Doligez, J. Garrigue, D. Rémy, and J. Vouillon. The Objective Caml system, documentation and user's manual (release 3.06). Tech. rep., INRIA, Rocquencourt, France, 2002.

[26] R. Manevich, G. Ramalingam, J. Field, D. Goyal, and M. Sagiv. Compactly representing first-order structures for static analysis. SAS (2002), LNCS 2477, Springer, 196-212.

[27] M. Martel. Static analysis of the numerical stability of loops. SAS (2002), LNCS 2477, Springer, 133-150.

[28] A. Miné. The octagon abstract domain library. http://www.di.ens.fr/ mine/oct/.

[29] A. Miné. A new numerical abstract domain based on difference-bound matrices. PADO (2001), LNCS 2053, Springer, $155-172$.

[30] A. Miné. The octagon abstract domain. IEEE AST in WCRE (2001), 310-319.

[31] M. Musuvathi, D. Park, A. Chou, D. Engler, and D. Dill. CMC: A pragmatic approach to model checking real code. UsENIX Association, OSDI 2002.

[32] S. Owre, N. Shankar, and D. Stringer-Calvert. PVS: An experience report. FM-Trends'98 (1999), LNCS 1641, Springer, 117-132.

[33] The Coq Development Team. The Coq proof assistant reference manual (version 7.4). Tech. rep., INRIA, Rocquencourt, France, 2003.

[34] M. Weiser. Program slicing. IEEE Transactions on Software Engineering SE-10, 4 (1984), 352-357. 